Original

\title{
Hypoxia-induced upregulation of angiogenic factors in immortalized human periodontal ligament fibroblasts
}

\author{
Takashi Kifune1), Hisanori (to' ${ }^{1)}$, Misa Ishiyama1), Satoko Iwasa1), Hiroki Takei'), \\ Tomokazu Hasegawa2), Masatake Asano ${ }^{3)}$, and Tetsuo Shirakawa ${ }^{1,4)}$ \\ 1)Department of Pediatric Dentistry, Nihon University School of Dentistry, Tokyo, Japan \\ 2)Department of Pediatric Dentistry, Tokushima University Hospital, Tokushima, Japan \\ 3)Department of Pathology, Nihon University School of Dentistry, Tokyo, Japan \\ 4)Division of Oral and Craniomaxillofacial Research, Dental Research Center, \\ Nihon University School of Dentistry, Tokyo, Japan
}

(Received November 20, 2017; Accepted December 18, 2017)

\begin{abstract}
Hypoxia induces complex cellular responses that are mediated by a key transcription factor, hypoxia-inducible factor-1 (HIF-1). HIF-1 promotes production of cytokines and angiogenic factors and contributes to recovery of injured tissues. In the present study, expressions of angiogenin (ANG) and vascular endothelial growth factor (VEGF), which are potent angiogenic factors in mammalian tissues, were examined in immortalized fibroblasts exposed to hypoxia. After $24 \mathrm{~h}$ of exposure to hypoxia, ANG and VEGF mRNAs expressions were significantly elevated in periodontal ligament (PDL) fibroblasts but not in embryonic fibroblasts. Hypoxia also increased productions of ANG and VEGF proteins in PDL fibroblasts. HIF-1 $\alpha$ mRNA expression was not affected by hypoxia in either fibroblast, although HIF-1 $\alpha$ protein expression was enhanced after exposure to hypoxia. Treatment of PDL fibroblasts with dimethyloxaloylglycine, a prolyl hydroxylase inhibitor that stabilizes the HIF-1 $\alpha$ protein, significantly increased expressions of ANG and VEGF mRNAs under normoxia. This suggests that stabilization of HIF-1 $\alpha$ is crucial for
\end{abstract}

Correspondence to Dr. Tetsuo Shirakawa, Department of Pediatric Dentistry, Nihon University School of Dentistry, 1-8-13 KandaSurugadai, Chiyoda-ku, Tokyo 101-8310, Japan

Fax:+81-3-3219-8353 E-mail: shirakawa.tetsuo@nihon-u.ac.jp

Color figures can be viewed in the online issue at J-STAGE.

doi.org/10.2334/josnusd.17-0441

DN/JST.JSTAGE/josnusd/17-0441 upregulation of ANG and VEGF in PDL fibroblasts. These results indicate that, under hypoxic conditions, HIF-1 $\alpha$ upregulates synthesis of ANG and VEGF in PDL fibroblasts and promotes angiogenesis.

Keywords: periodontal ligament fibroblasts; hypoxia; HIF-1; angiogenin; vascular endothelial growth factor.

\section{Introduction}

The periodontal ligament (PDL) is a soft connective tissue structure located between the cementum of the tooth root and alveolar bone (1). The PDL is formed predominantly by tooth root-specific fibroblasts, blood vessels, and collagen fibers. The fibroblasts produce various cytokines and vascular endothelial growth factor (VEGF), a protein that stimulates formation of blood vessels (2-6). VEGF is thought to contribute to homeostasis of microvasculature in the PDL. When periodontal tissue suffers traumatic injury, PDL cells aid in the recovery of damaged blood vessels through putative angiogenic pathway(s) involving the production of VEGF and other angiogenic factors.

Angiogenin (ANG), a member of the pancreatic ribonuclease (RNase) superfamily, is a potent angiogenic factor in mammalian tissues (7). ANG can stimulate microvascular endothelial cells to induce cell proliferation and formation of tubular structures (7-10). Retinal pigment epithelial cells respond to hypoxia by increasing ANG production, which causes overgrowth of blood 
Table 1 Primer sequences used for quantitation of gene expressions

\begin{tabular}{|c|c|c|}
\hline Gene & Sequences (F: forward, R: reverse) & GenBank number \\
\hline$A N G$ & $\begin{array}{l}\text { F 5' TGG AAC CCA TCT CCA GGA A 3' } \\
\text { R 5' GTG TGT GTA CCT GGA GTT ATC CTG A 3' }\end{array}$ & NM_001097577.2 \\
\hline$V E G F$ & $\begin{array}{l}\text { F 5' AGC TGC CTG ATG TCA ACT GCT TA 3' } \\
\text { R 5' GTT CAT TAC TGG AGC CCT GCA C 3' }\end{array}$ & NM_001025366 \\
\hline$S D F-1$ & $\begin{array}{l}\text { F 5' AGT CAG GTG GTG GCT TAA CAG 3', } \\
\text { R 5'AGA GGA GGT GAA GGC AGT GG 3' }\end{array}$ & NM_000609.6 \\
\hline$H I F-1 \alpha$ & $\begin{array}{l}\text { F 5', GTC TCG AGA TGC AGC CAG AT 3, } \\
\text { R 5, TCA CCA GCA TCC AGA AGT TTC 3, }\end{array}$ & NM_001243084.1 \\
\hline$\beta$-actin & $\begin{array}{l}\text { F 5', TGG CAC CCA GCA CAA TGA A 3, } \\
\text { R 5, CTA AGT CAT AGT CCG CCT AGA AGC A 3, }\end{array}$ & NM_001101.4 \\
\hline
\end{tabular}

vessels from the choroid of the eye in patients with progressive diabetes (10). A clinical study reported that ANG was present in gingival crevicular fluid collected from persons with chronic periodontitis (11). However, it is unknown whether PDL cells can produce ANG and, if so, how ANG production is influenced by hypoxia.

Hypoxia-inducible factor-1 (HIF-1) is a key oxygen sensor specifically activated during hypoxia (12). Both ANG and VEGF secretions were enhanced under hypoxic conditions in renal proximal tubular epithelial cells (13). HIF-1 acts on the production of ANG, VEGF, and many other bioactive factors by binding specific sites in the promoter regions of genes (13). In this study, the effects of hypoxia on ANG and VEGF expressions were investigated in human PDL fibroblasts (hPDLF) and embryonic fibroblasts (hEMBF) to clarify whether these fibroblasts are able to produce angiogenic factors under the control of HIF- $1 \alpha$.

\section{Materials and Methods \\ Cell lines and cell culture}

hPDLF (immortalized fibroblasts derived from human deciduous teeth; SH9) (14), and hEMBF (immortalized fibroblasts obtained from a human embryo; OUMS36T-1: JCRB cell bank) were used in this study. The properties of these cell lines have been described elsewhere $(14,15)$. hPDLF were maintained in $\alpha$-modified minimum essential medium ( $\alpha$-MEM, Wako, Tokyo, Japan) supplemented with $10 \%$ fetal bovine serum (FBS, Gibco, Carlsbad, CA, USA), and hEMBF were maintained in Dulbecco's modified Eagle's medium (DMEM, Gibco) supplemented with 10\% FBS. These cell cultures were maintained at $37^{\circ} \mathrm{C}$ in a $5 \% \mathrm{CO}_{2} / 95 \%$ air atmosphere (normoxic conditions). For mRNA experiments and western blotting, these cells were seeded onto 6-well dishes at $5.0 \times 10^{5}$ cells/well. For siRNA transfection experiments, the cells were seeded onto 24 -well dishes at $1.0 \times 10^{5}$ cells $/$ well.

\section{Exposure of fibroblasts to hypoxia}

Hypoxia was induced by transferring the cell culture dishes to an airtight jar containing AnaeroPack-Kenki 5\% (Mitsubishi Gas Chemical, Tokyo, Japan), a disposable $\mathrm{O}_{2}$-absorbing and $\mathrm{CO}_{2}$-generating agent that reduces the $\mathrm{O}_{2}$ concentration to less than $0.1 \%$ and maintains the $\mathrm{CO}_{2}$ concentration at about $5 \%$ (hypoxic conditions). The jar was placed in an incubator for $24 \mathrm{~h}$ at $37^{\circ} \mathrm{C}$. hPDLF and hEMBF cultured under normoxic conditions in a $\mathrm{CO}_{2}$ incubator were used as control.

\section{Real-time quantitative polymerase chain reaction analysis}

The mRNA expressions of ANG, VEGF, stromal cellderived factor-1 (SDF-1), and HIF-1 $\alpha$ were measured with a real-time quantitative polymerase chain reaction (RT-qPCR) assay. Total RNA was isolated from hPDLF and hEMBF cultured under normoxic (control) or hypoxic conditions for $24 \mathrm{~h}$ by using an RNeasy mini kit (QIAGEN, Hilden, Germany). RNA quality and concentration were assayed with a NanoDrop 2000 device (Thermo Fisher Scientific, Waltham, MA, USA). Purified total RNA was reverse-transcribed into cDNA by incubation with Recombinant RNase Inhibitor (TaKaRa, Tokyo, Japan), $5 \times$ Prime Script Buffer (TaKaRa), $5 \times$ Reverse Transcriptase M-MLV Buffer (TaKaRa), and Random Primer (Invitrogen, Carlsbad, CA, USA). The RT-qPCR assay was performed with SYBR Premix Ex Taq II (TaKaRa), and data analysis was done with a CFX96 Real-Time System (Bio-Rad, Hercules, CA, USA). For quantification of mRNA, the target expression level was normalized to the $\beta$-actin mRNA level. The primer sequences used for RT-qPCR are shown in Table 1.

\section{Western blotting}

Whole-cell lysates of hPDLF and hEMBF were prepared after $24 \mathrm{~h}$ of incubation under normoxic (control) or hypoxic conditions. The cells were harvested and lysed in cell lysis buffer $(2 \times$ TBS, Triton X-100). The protein 
concentration was measured by using Bio-Rad Protein Assay Dye Reagent Concentrate (Bio-Rad). Proteins of cell lysates were separated by electrophoresis in $10 \%$ SDS-polyacrylamide gels. The separated proteins were transferred to Immobilon PVDF membranes (Merck, Darmstadt, Germany) and blocked for $1 \mathrm{~h}$ in $1 \%$ BSAPBST. The membranes were then incubated overnight at $4^{\circ} \mathrm{C}$ with a rabbit anti-HIF- $1 \alpha$ antibody $(\times 1,000)$ (Novus Biologicals, Littleton, CO, USA) or a rabbit antiGAPDH antibody $(\times 1,000)$ (Santa Cruz Biotechnology, Dallas, TX, USA), followed by incubation with goat anti-rabbit IgG $(\mathrm{H}+\mathrm{L})$ secondary antibody $(\times 10,000)$ (Jackson Immuno Research, West Grove, PA, USA) for $1 \mathrm{~h}$ at room temperature. Specific protein bands on the membrane were detected by using Western ELC Substrate (Bio-Rad).

\section{Cytokine array analysis}

Angiogenic factors secreted into the medium by hPDLF under the culture conditions were analyzed by using a Human XL Cytokine Array Kit (R\&D Systems, Minneapolis, MN, USA). The cells were seeded onto 100-mm cell culture dishes at $1.0 \times 10^{6}$ cells/dish. Conditioned media were collected after 24-h incubation under normoxic (control) or hypoxic conditions and mixed with the reagents included in the assay kit. The mixed solution was reacted with the nitrocellulose membrane on which antibodies against angiogenic factors and cytokines had been spotted. After overnight incubation at $4^{\circ} \mathrm{C}$, the membrane was washed and reacted with Chemi Reagent Mix for $1 \mathrm{~min}$. Then, the protein signals on the membrane were visualized with the Universal Hood II system (Bio-Rad). The expression level of each cytokine was digitized with image analysis software (ImageQuant TL, GE Healthcare Life Sciences, Pittsburgh, PA, USA).

\section{Double immunofluorescence staining}

hPDLF were seeded on a chamber slide (Lab-Tek Chamber Slide; Thermo Fisher Scientific, Rochester, NY, USA) at $2.5 \times 10^{4}$ cells/dish. Subconfluent PDL cell monolayers were cultured under normoxic (control) or hypoxic conditions for $24 \mathrm{~h}$. The cell monolayers were fixed for $10 \mathrm{~min}$ at room temperature with $2 \%$ paraformaldehyde in PBS, permeabilized for $10 \mathrm{~min}$ at room temperature with $1 \%$ Triton-X100 diluted in PBS, blocked for $30 \mathrm{~min}$ at room temperature with $1 \%$ BSA-PBS, and incubated with a solution containing a mouse monoclonal anti-ANG antibody $(\times 100)($ Abcam, Cambridge, UK) and a rabbit polyclonal HIF-1 $\alpha$ antibody $(\times 50)$ (Novusbio, Littleton, CO, USA), or with a solution containing a mouse monoclonal anti-VEGF antibody $(\times 100)$ (Novusbio) and rabbit polyclonal HIF-1 $\alpha$ antibody $(\times 50)$ (Novusbio), for $12 \mathrm{~h}$ at $4^{\circ} \mathrm{C}$. Then, the cell monolayers were incubated for $60 \mathrm{~min}$ at room temperature with the appropriate combination of secondary antibodies. The secondary antibodies used were Alexa Fluor 546-conjugated donkey anti-mouse IgG (1:1,000; Invitrogen, Carlsbad, CA, USA) and Alexa Fluor 488-conjugated donkey anti-rabbit IgG (1:1000; Invitrogen). The stained cell monolayers were coverslipped with Fluoroshield Mounting Medium with DAPI (Abcam), which contained 4',6-diamidino-2-phenylindole (DAPI), and imaged by using a fluorescence microscope (Nikon Instruments, Tokyo, Japan) equipped with a $20 \times$ objective.

\section{SiRNA transfection}

To suppress HIF-1 $\alpha$ expression in hPDLF, a pre-designed siRNA was introduced by using a forward transfection method. $\alpha$-MEM used for maintaining the hPDLF culture was replaced with Opti-MEM-I Reduced Serum Medium (Thermo Fisher Scientific). After incubation for 30 min, the cells were washed and transfected with $2 \mu \mathrm{m}$ Stealth RNAi Pre-Designed siHIF-1 $\alpha$ (forward: 5'-CCA GCC GCU GGA GAC ACA AUC AUA U-3'; reverse: 5'-AUA UGA UUG UGU CUC CAG CGG CUG G-3') (Thermo Fisher Scientific) or $2 \mu \mathrm{m}$ Stealth RNAi siRNA Negative Control (Thermo Fisher Scientific) in the presence of Lipofectamine RNAiMAX Transfection Reagent (Thermo Fisher Scientific). After a 5-h incubation, the culture medium was replaced with $\alpha$-MEM supplemented with $10 \% \mathrm{FBS}$, and the cells were cultured for $24 \mathrm{~h}$ under hypoxic conditions. Then, RT-qPCR was used to analyze expressions of ANG and VEGF mRNAs.

\section{HIF-1 $\alpha$ stabilization with dimethyloxaloylglycine}

To evaluate the effects of HIF-1 $\alpha$ stabilization on expressions of ANG and VEGF mRNAs, hPDLF were cultured under normoxic conditions in $\alpha$-MEM supplemented with $1 \mathrm{mM}$ dimethyloxaloylglycine (DMOG, Enzo Life Sciences, Farmingdale, NY, USA), a competitive inhibitor of prolyl hydroxylase (PHD). Control hPDLF were cultured under normoxic conditions in $\alpha$-MEM that contained dimethyl sulfoxide (DMSO) but not DMOG. After a 24-h incubation, western blotting was used to evaluate the HIF-1 $\alpha$ protein level, and RT-qPCR was performed to evaluate expressions of ANG and VEGF mRNAs.

\section{Statistical analysis}

All data are presented as the mean \pm SEM. Statistical significance was determined by using the Student's $t$-test 

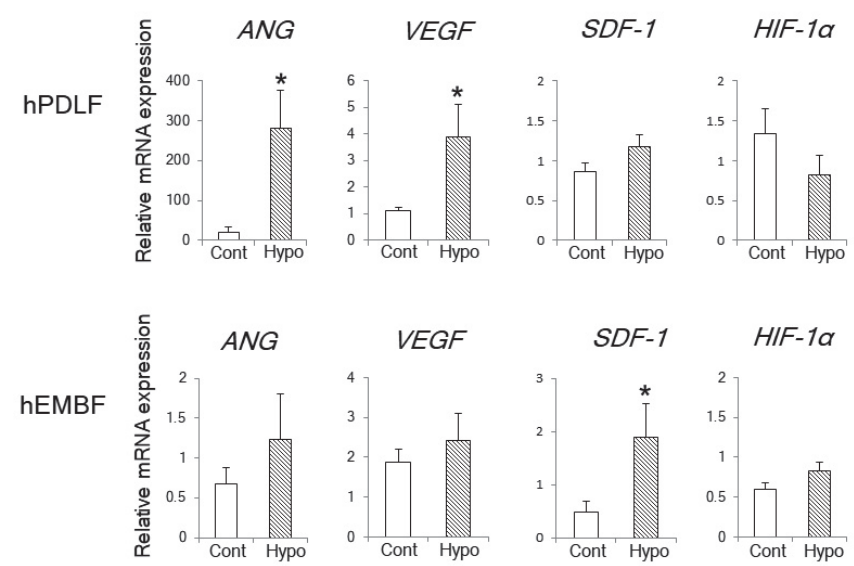

Fig. 1 Expression of ANG, VEGF, SDF-1, and HIF-1 $\alpha$ mRNAs in hPDLF and hEMBF incubated under normoxia (Cont) or hypoxia (Hypo). RT-qPCR analysis showed a significant increase in ANG and VEGF mRNA expression in hPDLF after $24 \mathrm{~h}$ of hypoxia. Under hypoxic conditions, SDF-1 mRNA expression increased in hEMBF but not in hPDLF. The data are presented as the mean \pm SEM. The asterisks indicate a significant difference in expression level, as compared with the control (Cont) group $(P<0.05$, Student's $t$-test).
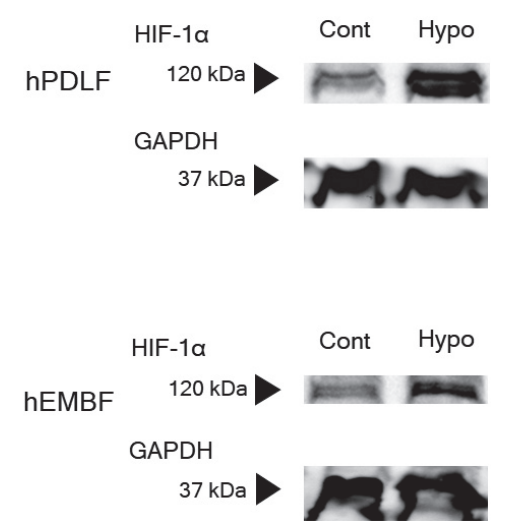

Fig. 2 Representative western blot images of HIF-1 $\alpha$ protein expression in hPDLF and hEMBF incubated under normoxia (Cont) or hypoxia (Hypo). HIF-1 $\alpha$ protein expression was enhanced in hPDLF and hEMBF after $24 \mathrm{~h}$ of hypoxia. GAPDH served as an internal control.

or Mann-Whitney $U$ test for pairwise comparisons. A $P$ value of $<0.05$ was considered to indicate statistical significance.

\section{Results}

mRNA expression in hPDLF and hEMBF exposed to hypoxia

Figure 1 shows the effects of hypoxia on expressions of mRNA in hPDLF and hEMBF. ANG and VEGF mRNA expression levels significantly increased in hPDLF cultured under hypoxic conditions $(P<0.05)$, but expressions of ANG and VEGF mRNAs in hEMBF were
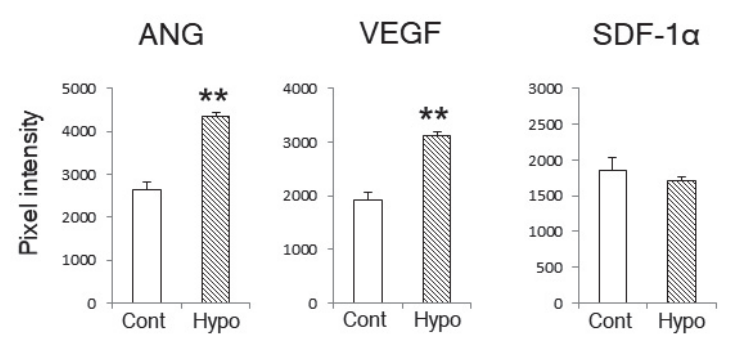

Fig. 3 Protein levels of ANG, VEGF, and SDF-1 secreted by hPDLF in culture media. ANG and VEGF levels were significantly higher in the culture media of hypoxia-exposed cells (Hypo) than in that of control cells (Cont). Pixel intensity corresponding to protein levels was analyzed with ImageQuant software. The data are presented as the mean \pm SEM. The double asterisks indicate a significant difference in pixel intensity, as compared with the control group $(P<0.001$, Student's $t$-test $)$.
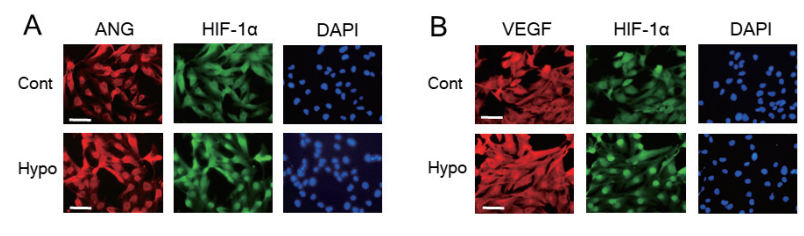

C
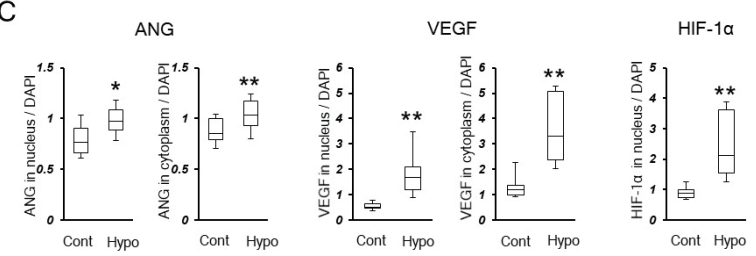

Fig. 4 Immunofluorescence staining of ANG, VEGF, and HIF-1 $\alpha$ in hPDLF exposed to normoxia (Cont) or hypoxia (Hypo). (A) ANG expression (red) in the cytoplasm and nucleus was enhanced after $24 \mathrm{~h}$ of hypoxia. (B) VEGF expression (red) in the cytoplasm and nucleus was enhanced under hypoxia. HIF-1 $\alpha$ expression was more prominent in the nucleus than in cytoplasm in hPDLF exposed to hypoxia (green). DAPI was used to label nuclear DNA of cells (blue). (C) Hypoxia significantly enhanced the immunofluorescence signals of ANG, VEGF, and HIF-1 $\alpha$ in hPDLF. The asterisks indicate a significant difference in intensity ratio, as compared with the control group $\left({ }^{*} P<0.01,{ }^{* *} P<0.001\right.$, Mann-Whitney $U$ test). Scale bar: $50 \mu \mathrm{m}$.

unaffected by hypoxia. In hPDLF and hEMBF, expression of HIF-1 $\alpha$ mRNA was unaffected by hypoxia, but SDF-1 mRNA was increased in hEMBF after exposure to hypoxia.

\section{HIF-1 $\alpha$ expression in hPDLF and hEMBF exposed to hypoxia}

HIF- $1 \alpha$ expression in hPDLF and hEMBF was compared between cultures exposed to normoxia or hypoxia for 24 h. Western blotting revealed that HIF-1 $\alpha$ expression was increased in hPDLF and hEMBF exposed to hypoxia (Fig. 2). 
A

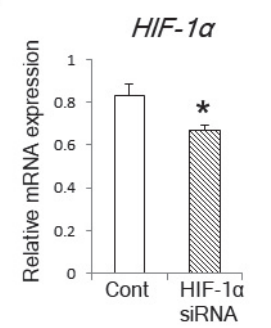

B
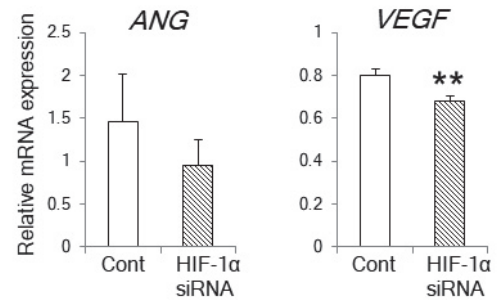

Fig. 5 Effects of HIF-1 $\alpha$ suppression with siRNA transfection in hPDLF. (A) Expression of HIF-1 $\alpha$ mRNA was reduced in hPDLF transfected with $2 \mu \mathrm{m}$ HIF- $1 \alpha$ siRNA and cultured under normoxic conditions for $24 \mathrm{~h}$. (B) Expression of ANG and VEGF mRNAs in hPDLF transfected with $2 \mu \mathrm{m}$ HIF-1 $\alpha$ siRNA was compared with that in control. The asterisks indicate a significant difference, as compared with the control group $\left({ }^{*} P<0.05,{ }^{* *} P<0.01\right.$, Student's $t$-test).

\section{Secretion of ANG, VEGF, and SDF-1 $\alpha$ from hPDLF exposed to hypoxia}

Protein levels of ANG, VEGF, and SDF-1 $\alpha$ in hPDLF culture media were evaluated by using a Human XL Cytokine Array. ANG and VEGF levels were significantly higher in culture media of hypoxia-exposed cells than in that of control cells $(P<0.001$, Student's $t$-test). SDF-1 $\alpha$ level was not affected by hypoxia (Fig. 3).

\section{Expression and localization of ANG, VEGF, and HIF-1 $\alpha$ in hPDLF}

Immunofluorescence staining for ANG, VEGF, and HIF- $1 \alpha$ revealed that hPDLF expressed these proteins in cytoplasm and the nucleus (Fig. 4A and B) and that ANG and VEGF were predominantly localized to cytoplasm (Fig. 4C). Hypoxia increased expressions of ANG and VEGF proteins in the cytoplasm and nucleus (Fig. 4C). HIF-1 $\alpha$ was moderately expressed in cytoplasm and the nucleus under normoxic conditions (Fig. 4A and B). However, HIF-1 $\alpha$ expression was much more obvious in the nucleus than in cytoplasm under hypoxic conditions.

\section{Effects of HIF-1 $\alpha$ siRNA on expressions of ANG and VEGF mRNAs in hPDLF}

First, the ability of a HIF- $1 \alpha$ siRNA to silence expression of HIF- $1 \alpha$ mRNA was tested in hPDLF. Treatment of
A

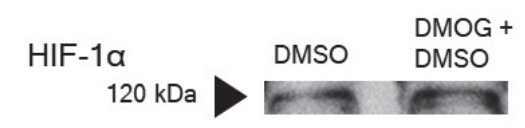

GAPDH

$37 \mathrm{kDa}$

B
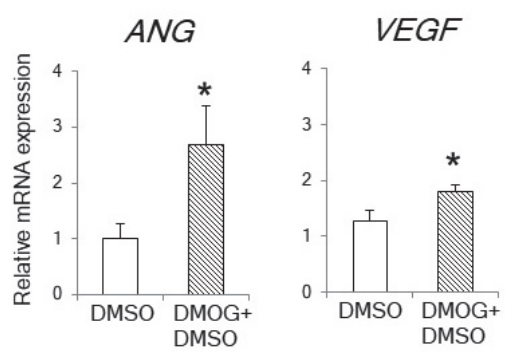

Fig. 6 Effects of DMOG on expression of HIF-1 $\alpha$ protein and ANG and VEGF mRNAs in hPDLF cultured under normoxic conditions. (A) Representative western blot images of HIF-1 $\alpha$ protein expression in hPDLF. Treatment of hPDLF with $1 \mathrm{mM}$ DMOG dissolved in DMSO for $24 \mathrm{~h}$ enhanced HIF-1 $\alpha$ expression. (B) RT-qPCR analysis showed that DMOG treatment upregulated ANG and VEGF mRNA expressions in hPDLF. The data are presented as the mean \pm SEM. The asterisks indicate a significant difference, as compared with the control group $(P<0.05$, Student's $t$-test).

hPDLF with an HIF- $1 \alpha$ siRNA at a dose of $2 \mu \mathrm{M}$ under normoxic conditions suppressed expression of HIF-1 $\alpha$ mRNA $(P<0.05)$ to a level approximately $80 \%$ that of control (Fig. $5 \mathrm{~A}$ ). Then the effects of $2 \mu \mathrm{M}$ HIF- $1 \alpha$ siRNA on the expressions of ANG and VEGF mRNAs were examined. Expression of VEGF mRNA was reduced by the HIF-1 $\alpha$ siRNA $(P<0.01)$, but expression of ANG mRNA was unaffected (Fig. 5B).

\section{Effects of DMOG on HIF-1 $\alpha$ protein and ANG and VEGF mRNA expressions in hPDLF}

Incubation of hPDLF in $\alpha$-MEM supplemented with 1 $\mathrm{mM}$ DMOG for $24 \mathrm{~h}$ increased HIF-1 $\alpha$ expression under normoxic conditions (Fig. 6A). As shown in Figure 6B, DMOG treatment significantly increased expressions of ANG and VEGF mRNAs in hPDLF $(P<0.05)$.

\section{Discussion}

This study examined the mechanism by which hypoxia upregulates expression of angiogenic factors in hPDLF. RT-qPCR and cytokine array analyses revealed that ANG and VEGF mRNAs and proteins were significantly upregulated under hypoxia in hPDLF but not in hEMBF. In contrast, mRNA expression of SDF-1, an angiogenic chemokine (16), was upregulated in hEMBF but not in hPDLF. Interestingly, SDF-1 $\alpha$, a protein produced 
via alternative splicing of the SDF-1 gene, was highly expressed in human PDL fibroblasts. Indeed, the expression level was much higher than that in human dermal fibroblasts (17). Hasegawa et al. reported that SDF-1 $\alpha$ expression was increased in hPDLF by treatment with transforming growth factor- $\beta 1$ but inhibited by treatment with basic fibroblast growth factor-2 (18), which suggests that fibroblasts exhibit cell type-specific reactivity to external stimuli.

ANG, VEGF, and SDF- $1 \alpha$ are potent angiogenic factors. ANG induces angiogenesis by activating endothelial cells in microvessels and triggers a number of biological processes, including cell migration, invasion, proliferation, and tubular structure formation (7-10). ANG binds receptors on the surface of endothelial cells, which facilitates its internalization and transport to the nucleolus (9). ANG is required for cell proliferation induced by various other angiogenic factors, including VEGF (19). Hypoxia increases ANG production in human malignant melanoma cells (20) and human dental pulp-derived cells (21). The present study is the first to show that hPDLF responds to hypoxia by increasing production of ANG and VEGF and that HIF-1 $\alpha$ has a pivotal role in activating $\mathrm{ANG}$ and VEGF production under hypoxic conditions.

A previous study reported that hypoxia enhanced HIF-1 $\alpha$ protein levels in PDL fibroblasts (22), which is consistent with our results regarding hPDLF. A recent study found that HIF- $1 \alpha$ mRNA expression was unchanged in PDL fibroblasts incubated for 8, 24, and 48 $\mathrm{h}$ under hypoxic conditions and was significantly higher only after $4 \mathrm{~h}$ incubation (23). Lai et al. reported that HIF- $1 \alpha$ mRNA expression in retinal pigment epithelial cells was significantly lower after 6,12 , and $24 \mathrm{~h}$ of exposure to hypoxia, while HIF-1 $\alpha$ protein expression in epithelial cells was significantly increased by hypoxia (10). In the present study, hypoxia upregulated expression of HIF-1 $\alpha$ protein but had no effect on HIF-1 $\alpha$ mRNA expression in hPDLF. These results strongly suggest that stabilization of HIF-1 $\alpha$ protein, rather than facilitation of HIF-1 $\alpha$ mRNA expression, is crucial for transcriptional activation of downstream genes under hypoxic conditions and that accumulation of HIF-1 $\alpha$ protein due to inactivation of PHD has a key role in this cascade (24-27). Upregulation of angiogenic cytokines in hPDLF induced by DMOG inhibition of PHD under normoxic conditions indicates that HIF-1 $\alpha$ stabilization is indispensable in increasing ANG and VEGF production in hPDLF.

After traumatic injury, hypoxia activates a variety of angiogenic growth factors and initiates neovascularization
(28). A previous study reported that the HeLa and Me180 cervical cancer cell lines and cervical cancer-derived fibroblasts released ANG and VEGF under normoxic conditions and that secretion of these angiogenic factors was significantly higher in fibroblasts than in tumor cells under hypoxic conditions (29). Immunofluorescence staining revealed that hypoxia increased expressions of ANG and VEGF protein in hPDLF in this study. In addition, both ANG and VEGF were predominantly localized to hPDLF cytoplasm, and hypoxia increased the intensity of cytoplasmic immunofluorescence under culturing conditions. This increase in ANG and VEGF immunofluorescence intensity under hypoxic conditions was concurrent with increases in secreted ANG and VEGF in culture media, as detected by the cytokine array.

The present study shows that hypoxia-induced upregulation of ANG and VEGF in hPDLF is dependent on the activity of HIF-1 protein, which survives hydroxylation by the oxygen-sensing enzyme PHD $(24,25)$ under hypoxic conditions. Evidence from some studies implicates putative HIF-1 signaling cascades in the inflammatory responses of human PDL fibroblasts (22-24,30-32). Although activation of the HIF-1 signaling cascade causes bone resorption by promoting production of inflammatory cytokines $(22,23,30-32)$, hypoxia-induced production of ANG and VEGF, which also depends on activation of the HIF-1 signaling cascade, may help hasten recovery from traumatic tissue damage.

\section{Acknowledgments}

This study was supported by a Grant-in-Aid for Scientific Research from the Japanese Society for the Promotion of Science (JSPS KAKENHI 26463120); a Health Labour Sciences Research Grant from the Ministry of Health, Labour and Welfare (10201010); MEXT-Supported Program for the Strategic Research Foundation at Private Universities 2010-2014 from the Ministry of Education, Culture, Sports, Science, and Technology, Japan; and research grants from the Dental Research Center and Sato Funds from Nihon University School of Dentistry.

\section{Conflict of interest}

The authors have no conflict of interest to declare.

\section{References}

1. Groeneveld MC, Everts V, Beertsen W (1995) Alkaline phosphatase activity in the periodontal ligament and gingiva of the rat molar: its relation to cementum formation. J Dent Res 74, 1374-1381.

2. Iizuka M, Yamauchi M, Ando K, Hori N, Furusawa Y, Itsukaichi H et al. (1994) Quantitative RT-PCR assay detecting the transcriptional induction of vascular endothelial growth factor under hypoxia. Biochem Biophys Res Commun 205, 1474-1480. 
3. Shimizu N, Ozawa Y, Yamaguchi M, Goseki T, Ohzeki K, Abiko Y (1998) Induction of COX-2 expression by mechanical tension force in human periodontal ligament cells. J Periodontol 69, 670-677.

4. Hasegawa T, Yoshimura Y, Kikuiri T, Yawaka Y, Takeyama S, Matsumoto A et al. (2002) Expression of receptor activator of NF-kappa B ligand and osteoprotegerin in culture of human periodontal ligament cells. J Periodontal Res 37, 405-411.

5. Yoshino H, Morita I, Murota SI, Ishikawa I (2003) Mechanical stress induces production of angiogenic regulators in cultured human gingival and periodontal ligament fibroblasts. J Periodontal Res 38, 405-410.

6. Miyagawa A, Chiba M, Hayashi H, Igarashi K (2009) Compressive force induces VEGF production in periodontal tissues. J Dent Res 88, 752-756.

7. Riordan JF, Vallee BL (1988) Human angiogenin, an organogenic protein. Br J Cancer 57, 587-590.

8. Moroianu J, Riordan JF (1994) Identification of the nucleolar targeting signal of human angiogenin. Biochem Biophys Res Commun 203, 1765-1772.

9. Wiedłocha A (1999) Following angiogenin during angiogenesis: a journey from the cell surface to the nucleolus. Arch Immunol Ther Exp 47, 299-305.

10. Lai K, Luo C, Zhang X, Ye P, Zhang Y, He J et al. (2016) Regulation of angiogenin expression and epithelial-mesenchymal transition by HIF- $1 \alpha$ signaling in hypoxic retinal pigment epithelial cells. Biochim Biophys Acta 1862, 1594-1607.

11. Sakai A, Ohshima M, Sugano N, Otsuka K, Ito K (2006) Profiling the cytokines in gingival crevicular fluid using a cytokine antibody array. J Periodontol 77, 856-864.

12. Melvin A, Rocha S (2012) Chromatin as an oxygen sensor and active player in the hypoxia response. Cell Signal, 24, $35-43$.

13. Nakamura M, Yamabe H, Osawa H, Nakamura N, Shimada M, Kumasaka R et al. (2006) Hypoxic conditions stimulate the production of angiogenin and vascular endothelial growth factor by human renal proximal tubular epithelial cells in culture. Nephrol Dial Transplant 21, 1489-1495.

14. Sakaguchi M, Miyazaki M, Inoue Y, Tsuji T, Kouchi H, Tanaka $\mathrm{T}$ et al. (2000) Relationship between contact inhibition and intranuclear S100C of normal human fibroblasts. J Cell Biol 149, 1193-1206.

15. Hasegawa T, Chosa N, Asakawa T, Yoshimura Y, Ishisaki A, Tanaka M (2010) Establishment of immortalized human periodontal ligament cells derived from deciduous teeth. Int J Mol Med 26, 701-705.

16. Salcedo R, Oppenheim JJ (2003) Role of chemokines in angiogenesis: CXCL12/SDF-1 and CXCR4 interaction, a key regulator of endothelial cell responses. Microcirculation 10, 359-370.

17. Yashiro Y, Nomura Y, Kanazashi M, Noda K, Hanada N, Nakamura Y (2014) Function of chemokine (CXC motif) ligand 12 in periodontal ligament fibroblasts. PLoS One 9, e95676.

18. Hasegawa T, Chosa N, Asakawa T, Yoshimura Y, Fujihara Y, Kitamura $T$ et al. (2012) Differential effects of TGF- $\beta 1$ and FGF-2 on SDF-1 $\alpha$ expression in human periodontal ligament cells derived from deciduous teeth in vitro. Int J Mol Med 30, 35-40.

19. Kishimoto K, Liu S, Tsuji T, Olson KA, Hu GF (2005) Endogenous angiogenin in endothelial cells is a general requirement for cell proliferation and angiogenesis. Oncogene 24, 445-456.

20. Hartmann A, Kunz M, Köstlin S Gillitzer R, Toksoy A, Bröcker EB et al. (1999) Hypoxia-induced up-regulation of angiogenin in human malignant melanoma. Cancer Res 59, 1578-1583.

21. Janjić K, Edelmayer M, Moritz A, Agis H (2017) L-mimosine and hypoxia can increase angiogenin production in dental pulp-derived cells. BMC Oral Health 17, 87.

22. Park HJ, Baek KH, Lee HL, Kwon A, Hwang HR, Qadir AS et al. (2011) Hypoxia inducible factor-1 $\alpha$ directly induces the expression of receptor activator of nuclear factor- $\mathrm{kB}$ ligand in periodontal ligament fibroblasts. Mol Cells 31, 573-578.

23. Gölz L, Memmert S, Rath-Deschner B, Jäger A, Appel T, Baumgarten $G$ et al. (2015) Hypoxia and P. gingivalis synergistically induce HIF-1 and NF- $\mathrm{kB}$ activation in PDL cells and periodontal diseases. Mediators Inflamm doi: $10.1155 / 2015 / 438085$.

24. Agis H, Watzek G, Gruber R (2012) Prolyl hydroxylase inhibitors increase the production of vascular endothelial growth factor by periodontal fibroblasts. J Periodontal Res 47, 165-173.

25. Ogle ME, Gu X, Espinera AR, Wei L (2012) Inhibition of prolyl hydroxylases by dimethyloxaloylglycine after stroke reduces ischemic brain injury and requires hypoxia inducible factor-1 $\alpha$. Neurobiol Dis 45, 733-742.

26. Ding H, Chen S, Song WQ, Gao YS, Guan JJ, Wang Y et al. (2014) Dimethyloxaloylglycine improves angiogenic activity of bone marrow stromal cells in the tissue-engineered bone. Int J Biol Sci 10, 746-756.

27. Zhdanov AV, Okkelman IA, Collins FW, Melgar S, Papkovsky DB (2015) A novel effect of DMOG on cell metabolism: direct inhibition of mitochondrial function precedes HIF target gene expression. Biochim Biophys Acta 1847, 1254-1266.

28. Payen VL, Brisson L, Dewhirst MW, Sonveaux P (2015) Common responses of tumors and wounds to hypoxia. Cancer J 21, 75-87.

29. Pilch H, Schlenger K, Steiner E, Brockerhoff P, Knapstein P, Vaupel P (2001) Hypoxia-stimulated expression of angiogenic growth factors in cervical cancer cells and cervical cancer-derived fibroblasts. Int J Gynecol Cancer 11, 137-142.

30. Motohira H, Hayashi J, Tatsumi J, Tajima M, Sakagami H, Shin K (2007) Hypoxia and reoxygenation augment boneresorbing factor production from human periodontal ligament cells. J Periodontol 78, 1803-1809.

31. Jiang C, Li C, Ren Y, He Y, Li Y, Feng X et al. (2014) Hypoxia augments lipopolysaccharide-induced cytokine expression in periodontal ligament cells. Inflammation 37, 1413-1423.

32. Yu XJ, Xiao CJ, Du YM, Liu S, Du Y, Li S (2015) Effect of hypoxia on the expression of RANKL/OPG in human periodontal ligament cells in vitro. Int J Clin Exp Pathol 8, $12929-12935$. 